ISSN: 2602-8506

Vol. 3, N4., p.20-43, octubre - diciembre, 2019

Recibido: 03-08-2019 /Aceptado:06-09-2019/ Publicado: 05-10-2019

\title{
Impulso a la productividad y competitividad de los emprendedores de la ciudad del Tena para el crecimiento económico sustentable
}

Boosting the productivity and competitiveness of entrepreneurs in the city of Tena for sustainable economic growth

Edison Becerra Molina. ${ }^{1}$, Glenda Ramón Poma. ${ }^{2}$, Estefanía Venegas Sánchez. ${ }^{3}$ \& Pedro Astudillo Arias. ${ }^{4}$

\begin{abstract}
This article is based on the real needs of entrepreneurs who promote the entrepreneurial spirit and sustainability of small and medium enterprises in the city of Tena, in that sense many of the businesses have some shortcomings, one of the most important is the need training of merchants and entrepreneurs in different areas of small business, the objective of this research is to see how the improvement of the skills and abilities of entrepreneurs impacted, considering the application and transfer of knowledge, for this purpose with the participation of the students of the UCACUE, the same ones that carried out workshops and training accompaniments to strengthen the productive entrepreneurship of the microentrepreneurs who carry out trade activities in the city markets.

The methodology used in the research was descriptive, based on the bibliographic and documentary review, and the survey instrument, which sought to diagnose and punctuate the needs of entrepreneurs.

For the calculation of the sample size, the formula of the standard error of the population has been used, corresponding to 148 surveyed merchants, the results showed microentrepreneurs trained in administration techniques, finance, marketing, customer service, among others. and evaluated factors that influence entrepreneurship and the
\end{abstract}

\footnotetext{
${ }^{1}$ Universidad Católica de Cuenca, Ecuador, jbecerram@ucacue.edu.ec

${ }^{2}$ Universidad Católica de Cuenca, Cuenca, Ecuador, gramon@ucacue.edu.ec

${ }^{3}$ Universidad Católica de Cuenca, Cuenca, Ecuador, nvenegass@ucacue.edu.ec

${ }^{4}$ Universidad Católica de Cuenca, Cuenca, Ecuador, pastudillo@ucacue.edu.ec
} 
importance of components that affect sustainability, the generation of wealth, employment and the revitalization of the economy, which ultimately ends up benefiting society.

Keywords: University, connection, entrepreneurship, micro-enterprise, accompaniment.

\section{Resumen}

Este artículo está basado en las necesidades reales de los emprendedores que promueven el espíritu empresarial y la sostenibilidad de los pequeños y medianas empresas de la ciudad del Tena, en ese sentido muchos de los negocios presentan algunas falencias, una de las más importantes es la necesidad de capacitación de los comerciantes y emprendedores en las distintas áreas de la pequeña empresa, el objetivo de la presente investigación estriba en ver como el perfeccionamiento de las competencias y habilidades de los emprendedores impactó, considerando la aplicación y transferencia del conocimiento, para ello se contó con la participación de los alumnos de la UCACUE, los mismos que efectuaron talleres y acompañamientos de capacitación para fortalecer el emprendimiento productivo de los microempresarios que realizan actividades de comercio en los mercados de la ciudad.

La metodología utilizada en la investigación fue de campo de carácter descriptivo, en base a la revisión bibliográfica y documental, y al instrumento la encuesta, que buscó diagnosticar y puntualizar las necesidades de los emprendedores.

Para el cálculo del tamaño de la muestra se ha utilizado la fórmula del error estándar de la población, correspondiendo a 148 comerciantes encuestados, los resultados arrojaron microempresarios capacitados en técnicas de administración, finanzas, marketing, servicio al cliente, entre otros., además se medió y evaluó factores que influyen en el emprendimiento y la importancia de componentes que inciden en la sostenibilidad, la generación de riqueza, empleo y la dinamización de la economía, lo que finalmente, termina en beneficio a la sociedad.

Palabras claves: Universidad, vinculación, emprendimiento, microempresa, acompañamiento.

\section{Introducción}

En América Latina el impulso a la actividad emprendedora se ha transformado en una de las principales políticas, de allí que las microempresas y las pequeñas empresas tienen una 
Vol. 3, N4., p.20-43, octubre - diciembre, 2019

representatividad del $80 \%$ del total de la economía, según Zamora (2011). Ecuador es uno de los países más emprendedores de Latinoamérica. Sin embargo, la curva de emprendimiento decae a tan solo seis meses, Así lo señala el estudio Global Entrepreneurship Monitor GEM Ecuador (2014), el análisis indico que el incremento del emprendimiento se da por necesidad; es decir, por falta de otras opciones de ingresos. En consecuencia, el estudio determinó que el 22,7\% de los adultos afirmó haber emprendido aprovechando una oportunidad en el mercado, mientras que el 12,1\% lo hizo por necesidad. Con esas cifras, Ecuador pasó a tener la tasa de emprendimientos por necesidad más alta de la región, seguido de Perú $(5,25 \%)$ y Brasil $(4,95 \%)$.

En el presente caso el emprendimiento productivo que se ha generado en la ciudad de San Juan de los dos ríos de Tena, como consecuencia de las necesidades experimentadas por sus habitantes y la posibilidad de ser satisfechas por personas emprendedoras de la localidad, ha permitido la generación de negocios y empresas que han permitido la dinamización de la economía de este cantón.

Tena es una ciudad ecuatoriana; cabecera cantonal del Cantón Tena y capital de la Provincia de Napo, así como la urbe más grande y poblada de la misma. Se localiza al centronorte de la Región amazónica del Ecuador, en los flancos externos de la cordillera oriental de los Andes, atravesada por los ríos Tena y Pano, a una altitud de $510 \mathrm{msnm}$ y con un clima lluvioso tropical de $25^{\circ} \mathrm{C}$ en promedio; para el año 2019 cuenta con una población proyectada de 77.502 habitantes; los que de acuerdo a las estadísticas publicadas por el INEC, 39.448 son hombres y 38.054 mujeres; 18.540 del sexo masculino y 12.557 del femenino constituyen la población económicamente activa del cantón; del PEA de hombres, 2.002 se dedican a la actividad de servicios y vendedores y de mujeres 2.147 se desenvuelven en trabajos similares $\mathrm{y}$ en actividades agrarias.

El grupo humano que se dedica al comercio y la prestación de servicios en los principales mercados de la ciudad del Tena, tienen una formación educativa de nivel básico y un porcentaje reducido con bachillerato; esta realidad ha motivado para que estudiantes de la Universidad Católica de Cuenca, de la carrera de Administración de Empresas, bajo la modalidad a distancia, hayan planificado, organizado y ejecutado talleres de capacitación sobre administración, finanzas, tributación, recursos humanos y marketing; prevención y control de incendios, 
primeros auxilios básicos, normas de higiene y sanidad en preparación de alimentos; manejo de la imagen del negocio, servicio al cliente, prevención, legislación y normativa de seguridad y salud ocupacional, prevención al uso y consumo de sustancias en espacios laborables; y, señalética y seguridad.

Estas actividades que se realizaron para dar cumplimiento con la vinculación con la sociedad, tienen como propósito mejorar las condiciones de vida de los emprendedores, haciendo que los emprendimientos sean eficientes generando bienes y servicios de calidad que satisfagan de mejor manera las necesidades de los clientes.

La presente investigación tiene como finalidad identificar las necesidades reales de capacitación que requirieron los emprendedores de los mercados de la ciudad del Tena, y determinar el impacto tuvieron estas asesorías, acompañamientos y capacitaciones, de manera pues que sepamos de buena tinta de qué manera las competencias específicas y genéricas son necesarias para desempeñarse de manera eficiente dentro de su propio emprendimiento, de allí se deduce que los comerciantes y empresarios, a través de la realización de eventos de capacitación contribuyeron al crecimiento micro empresarial, incrementando su rentabilidad y siendo competitivos en el mercado.

Igualmente, los microempresarios en la actualidad afrontan cada vez más incertidumbre que genera, un mundo cada vez más globalizado y cambiante, haciéndose por tanto necesario nuevas herramientas, conceptos, información o procesos, que se convierten en temas de interés, haciéndose necesario conocer y adaptarse a estas nuevas sinergias, toda vez que son necesarios y transcendentales para poder ser competitivos en la localidad, la región, el país y por qué no decirlo a nivel mundial.

\section{Metodología}

El tipo de investigación está ligado con el diseño de campo y el nivel descriptivo, esgrimiendo técnicas cualitativas y cuantitativas de recolección de datos, como lo son la encuesta que según Sampieri (2010) es: Aquella que busca especificar propiedades, características y rasgos importantes de cualquier fenómeno que se analice. Describe tendencias de un grupo o población; respaldadas en el apoyo de la revisión bibliográfica y documental de registros e informes de la empresa pública de desarrollo productivo y competitividad, "Empudepro-Tena EP”, por cuanto 
ISSN: 2602-8506

Vol. 3, N4., p.20-43, octubre - diciembre, 2019

mediante el proyecto de vinculación se destaca el desarrollo de la cultura emprendedora en los estudiantes de la Universidad Católica de Cuenca, generando acciones orientadas al aporte del micro emprendimiento, como un eje transversal del proceso académico.

Para la elaboración del Proyecto se utilizó la metodología Inductiva para diagnosticar la situación de los comerciantes de los mercados en temas de comercialización, producción, transporte, distribución, recursos humanos, temas tributarios, servicio al cliente, venta de los productos, salud ocupacional, primeros auxilios, seguridad y prevención de incendios, entre otros, luego se diseñó un modelo integral de asesoría y acompañamiento para los comerciantes del cantón Tena de los mercados Central y Sur y mercado de Misahualli, para luego mediante nuestro investigación y la utilización del instrumento de la encuesta proceder evaluar los resultados e impactos del proyecto a corto, mediano y largo plazo, y propiciar la correcta aplicación de los conocimientos adquiridos en su quehacer diario de producción, transporte, distribución y venta de los productos en los mercados.

En el cálculo del tamaño de la muestra se ha utilizado la fórmula del error estándar de la población, debido a que es una investigación de tipo social con una población finita. Además; al fijar el tamaño de la muestra o número de encuestas se ha tenido cuidado en definir una muestra lo suficientemente grande y representativo del universo de tal manera que se pueda generalizar los resultados de la muestra a toda la población estudiada, de la población de 340 microempresarios registrados entre comerciantes formales e informales, se realizó una investigación de carácter descriptivo, en base a la revisión bibliográfica y documental de registros e informes de la empresa pública de desarrollo productivo y competitividad, "Empudepro-Tena EP", así como del proyecto de vinculación denominado "Fortalecimiento al emprendimiento productivo e incubación de los microempresarios de los mercados de la ciudad de Tena”. Los resultados de la investigación de una muestra de 148 emprendedores, indicaron que las necesidades de capacitación que mayormente requirieron los comerciantes y empresarios, fueron beneficiosas y que han permitido contribuir de esta forma al desarrollo económico de los sectores sociales de la provincia de Napo, para ello se consideraron dos variables relevantes dentro de la metodología de la presente investigación como es el emprendimiento y la sostenibilidad. 


\section{Fundamentación Teórica}

Consideramos dentro de nuestra investigación es importante conocer diversos puntos de vista sobre lo que significa el concepto de capacitación, al respecto según, Werther \& Davis (2011) la necesidad de capacitación surge cuando hay diferencia entre lo que una persona debería saber para desempeñar una tarea, y lo que realmente sabe, por ello su importancia radica principalmente en los beneficios que genera, que de acuerdo a Romero (2010), la importancia de la capacitación toda vez que ayuda al microempresario a lograr a una mayor rentabilidad, mejora el conocimiento de sus actividades empresariales, eleva la moral de la fuerza de trabajo, crea mejor imagen, mejora la relación con sus clientes y subordinados cuando existen empleados, además de contribuir a la formación de líderes, fomenta talantes hacia el logro de los objetivos personales y micro empresariales.

Según, Calderón (2018), la capacitación en el trabajo, la conforman diversas actividades enfocadas a desarrollar habilidades y mejorar actitudes del personal respecto a las tareas que realizan, ayudan al individuo, permite que el trabajador interiorice y ponga en práctica las variables de motivación, realización, crecimiento y progreso, alimenta la confianza, posición asertiva y el desarrollo, contribuye positivamente en el manejo de conflictos y tensiones, sube el nivel de satisfacción de los clientes y permite el logro de metas individuales. Por consiguiente, entendemos que un requerimiento o necesidad de capacitación es cuando una persona identifica la carencia de algún discernimiento o comprensión, de allí que requiere de una capacitación o asesoría para entender, completar o ampliar sus conocimientos y habilidades ineludibles, para el desempeño de una mejor manera una tarea, función o actividad.

En lo referente a las áreas de acompañamiento, talleres, asesorías y sesiones de trabajo de acuerdo a nuestros resultados obtenidos se determinaron encuestas contrastando con entrevistas semiestructuradas, realizadas a seis microempresarios del mercado de Misahualli, ciento ocho del mercado sur y treinta y cuatro del mercado central, dando un total de la muestra 148 emprendedores, el análisis indicó que se asocian con lo expuesto por el estudio de Lituma (2017), donde establece que mayoritariamente los comerciantes desean capacitarse para el mejoramiento de su negocio en ventas, inversiones, administración, servicio al cliente, al igual que su necesidad por instruirse en aspectos tributarios, por no tener el conocimiento de cómo 
ISSN: 2602-8506

Vol. 3, N4., p.20-43, octubre - diciembre, 2019

realizar los trámites correspondientes a dicha área, otro ejemplo básico, podría ser, los aspectos que se utilizan para fijar el precio de la mercadería, que para el estudio de Argos (2016) el 66\% de los participantes de la investigación manifiestan que el criterio principal para determinar los costos es basado en el precio de la competencia, y en menores proporciones a los costos del producto y la situación económica de los clientes, de tal manera que podemos considerar a todas estas áreas como fundamentales para el correcto funcionamiento de sus negocios.

Así mismo, históricamente el sector empresarial ha estado presente en las actividades humanas, contribuyendo al desarrollo económico de los sectores sociales por medio de la generación de riqueza, empleo y la dinamización de la economía al introducir una nueva unidad de negocio, lo que finalmente, termina beneficiando a toda la sociedad.

Es oportuno indicar que la educación empresarial empieza a tener un papel fundamental en los campos de estudios universitarios, puesto que la estadística del informe del Banco Mundial (2014), sostiene que se crean nuevas empresas cada vez con mayor celeridad. No obstante, la quiebra y cierre de nuevas empresas también es alta. De allí la trascendencia de una buena educación empresarial, puesto que varios autores como Drucker, Schumpeter, Stevenson, Aronsson, Arieu, entre otros, contribuyen con sus estudios al éxito de los empresarios; y el afán fue contribuir una formación relacionada.

La presente investigación tiene relevancia científica por cuanto permitió contribuir con los postulados del Plan Nacional de Desarrollo para el Buen Vivir 2017- 2021 "Toda una Vida" (2017), el eje 2 que esta representa, hace referencia a la economía al servicio de la sociedad, por cuanto el sistema económico es social y solidario. La economía está al servicio de la sociedad para garantizar los derechos y en ella se articulan los subsistemas públicos, privado, y popular; y los tres requieren estímulos y regulación normativa del Estado, como es lógico el propósito es contribuir, según el objetivo cinco del citado plan "Impulsar la productividad y competitividad para el crecimiento económico sustentable de manera redistributiva y solidaria". (p.35).

Según la Política 5.3 Plan Nacional para el Buen Vivir 2017-2021 (2017), es importante destacar que esta política tiene como objeto "Promover la investigación, la formación, la capacitación, el desarrollo y la transferencia tecnológica, la innovación y el emprendimiento, en 
articulación con las necesidades sociales, para impulsar el cambio de la matriz productiva" (p.74).

De igual manera, la iniciativa empresarial para el desarrollo de acuerdo a la resolución aprobada por la Agenda 2030 y los Objetivos de Desarrollo Sostenible (2016) en América Latina y el Caribe, Promueve políticas orientadas al desarrollo que apoyen las actividades productivas, la creación de puestos de trabajo decentes, el emprendimiento, la creatividad y la innovación, y fomentar la formalización y el crecimiento de las microempresas y las pequeñas y medianas empresas, incluso mediante el acceso a servicios financieros.

Por su parte, un comerciante es la persona que se dedica habitualmente al trabajo que también ayuda en la economía. También se denomina así al propietario de un comercio. En este sentido, son comerciantes las personas que, de manera habitual, se ocupan en alguna de las actividades que la ley considera mercantiles (actos de comercio). La habitualidad constituye un elemento esencial de la definición: no toda persona que realice un acto de comercio ocasional (por ejemplo, quien compra en una tienda) se constituye en comerciante, sino que sólo es considerado comerciante desde la perspectiva del Derecho Mercantil quien se dedique al comercio de forma habitual, es importante definir la forma en la que se produce y hacia dónde va a estar destinada dicha producción, de allí que la transformación de la matriz productiva está basada en cuatro ejes que son la diversificación productiva, agregar valor a la producción, sustitución de importaciones y fomentar las exportaciones de bines de consumo final, por ello que hemos centrado nuestra investigación en los comerciantes del Cantón Tena que pertenecen a la provincia de Napo, porque tienen la calidad de emprendedores.

Siguiendo la misma línea en el proceso de indagación, aquí se abordaron investigaciones en relación con el objeto de estudio en el ámbito nacional y local que precisan su enfoque donde se aborda la conceptualización de la informalidad, que es una de los factores que obstaculizan las actividades de emprendimiento.

En relación a este aspecto, Pacek (2008), manifiesta que la informalidad se presenta mayoritariamente en los países en vías de desarrollo y entre las principales causales están: las pocas oportunidades laborales; la falta de niveles de educación, y el problema para acceder a una línea de crédito en el sector cooperativo y/o financiero. 
Vol. 3, N4., p.20-43, octubre - diciembre, 2019

Todo lo descrito en este tema sobre la problemática, hace pensar que ésta forma de hacer comercio genera desórdenes en los mercados, que deben ser de interés primario para las autoridades del GAD Municipal mediante la toma de decisiones eficientes y concretas, orientadas a ir disminuyendo este grave problema social.

Consecuentemente, se llega a la conclusión que "las personas con limitados niveles de educación, debido a la necesidad de subsistir y la responsabilidad que mantiene con su familia y de sentirse útil para la sociedad, se dedican a diversas actividades a través de las cuales obtienen recursos económicos, sin considerar el esfuerzo que conlleva la consecución de este propósito". Por lo manifestado, de acuerdo a información primaria recopilada, se puedo aseverar que, este sector de la población genera limitados ingresos para cubrir las necesidades básicas de sus hogares, por tanto, se podría considerar como aquel que vive en la pobreza.

Para este análisis se consideraron los mercados específicamente del cantón el Tena, durante los días de feria se da una gran afluencia de la ciudadanía en los distintos mercados: Mercado Central, Mercado Sur (Feria Libre), Mercado de Misahuallí, es cuando aprovechan los comerciantes informales para realizar actividades de comercio, en unos casos expendiendo productos de su propia producción o mediante la compra a los mayoristas para volverlos a vender y, como no cuentan con el respectivo carnet de autorización, no tienen un puesto específico, se ubican a los alrededores de los mercados o en los lugares de acceso al interior, son de fácil visibilidad para ser perseguidos por los Policías municipales, dando lugar a enfrentamientos.

Todo lo descrito en este tema sobre la problemática, hace pensar que ésta forma de hacer comercio genera desórdenes en los mercados, que deben ser de interés primario para las autoridades del GAD Municipal del Cantón el Tena, mediante la toma de decisiones eficientes y concretas, orientadas a ir disminuyendo este grave problema social.

En esta orientación, la información que aportó al estudio en referencia permitió establecer que mediante el proyecto de Vinculación con la Sociedad denominado "Fortalecimiento al emprendimiento productivo e incubación de los microempresarios de los mercados de la ciudad de Tena", coadyuvó para solucionar la carencia de un modelo de gestión productiva, administrativa, contable, servicio al cliente y tributaria, así como, la inexistencia de un plan de 
marketing que oriente el proceso de comercialización y ventas, la información recogida permitió determinar también que el problema se originó también en el desconocimiento de los comerciantes de herramientas eficaces de gestión gerencial y se agudiza en el desempeño diario de la actividad productiva, conglomerados todos estos componentes en un plan de negocio, que no más que una declaración formal de un conjunto de objetivos micro empresariales.

Así mismo, al no existir el control estadístico del proceso y rigurosos sistemas de control de calidad, la producción no tuvo horizonte. Igualmente, en cuanto a lo administrativo, contable y tributario, los comerciantes en muchos casos no saben cuánto gana o cuánto pierde, no dispone de una fotografía situacional.

Es conveniente destacar que el análisis de la presente investigación, tuvo como objetivo primordial promover al sector micro empresarial emprendedor de esta parte del país, para lograr el desarrollo como medio para mejorar procesos eficientes de distribución del ingreso y generación de empleo, y que por su puesto luego de la medición del impacto de estas capacitaciones se estableció en los comerciantes la mejoría de actitud, autoestima entre otros, pero sobre todo en materia de organización, administración, finanzas, tributación, servicio al cliente, manejo de residuos sólidos, emprendedores que además mejoraron la imagen de los mercados y garantizaron la salud de los consumidores en cuanto a la manipulación de alimentos, productos y servicio.

\section{Resultados}

El emprendimiento al igual que la creación de empresas se ha convertido en un tema de interés mundial por su aporte al desarrollo económico y social de una región. Es por ello, que la educación para la formación empresarial es una actividad importante en los programas formativos de los jóvenes en sus carreras de pregrado.

De acuerdo a esta conceptualización, la propuesta curricular básica de acompañamiento a miembros de los mercados de la ciudad del Tena, según nuestro estudio, hace necesario destacar la participación activa que tuvieron los estudiantes de la carrera de Administración de Empresas, Modalidad a Distancia de la Universidad Católica de Cuenca, quienes aportaron para la consecución del proyecto mediante acompañamientos, sesiones de trabajo y talleres en materia de administración, finanzas, tributación, producción, control de calidad, investigación 
ISSN: 2602-8506

Vol. 3, $\mathrm{N}^{\circ} 4 .$, p.20-43, octubre - diciembre, 2019

de mercados y marketing, accidentes de trabajo y control y prevención de incendios, primeros auxilios, normas de higiene, manejo de la imagen del negocio, servicio al cliente, hasta el ciclo marzo- agosto 2019, promoviendo así la importancia del sector micro empresarial de la ciudad de Tena, contribuyendo así al desarrollo como medio para adelantar procesos eficientes de distribución del ingreso y generación de empleo.

Es por ello que de acuerdo al proyecto al proyecto de vinculación denominado "Fortalecimiento al emprendimiento productivo e incubación de los microempresarios de los mercados de la ciudad de Tena", realizados por los estudiantes de la Unidad Académica de Administración de la UCACUE, además de la revisión bibliográfica y documental de registros e informes de la empresa pública de desarrollo productivo y competitividad, "Empudepro-Tena EP”, tuvieron un rol sobresaliente en la investigación formativa, cabe destacar que los acompañamientos, talleres, asesorías y resultados de sesiones de trabajo para mejorar la organización y el ordenamiento de los mercados del Tena, se efectuaron en el siguiente orden de temáticas, asistencia y participaciones.

El análisis indicó que se ha asesorado a 284 emprendedores, mediante sesiones de trabajo y talleres en temas referentes a la administración, producción, control de calidad, investigación de mercados y marketing, que es la que mayor aceptación ha tenido por los pequeños comerciantes, seguido de las capacitaciones en normativa técnico-legal en prevención de riesgos laborales en el Ecuador con 248 adiestramientos, donde han tenido activa participación los funcionarios del Instituto Ecuatoriano de Seguridad Social, y estudiantes de la UCACUE, con su proyecto de vinculación con la comunidad, seguido con 242 capacitaciones de prevención al uso y consumo de sustancias en espacios laborables a los comerciantes de los mercados con el apoyo del Ministerio de Salud Pública, y la Secretaria Técnica de Drogas- SETAD, posteriormente con 229 asesorías tenemos servicio al cliente, dictadas por estudiantes de la UCACUE, en menor volumen de capacitaciones con 220 prevención y control de incendios, 214 manejo de la imagen del negocio, 186 en normas de higiene y sanidad en preparación de alimentos, con 172 Señalética y seguridad; y por último con 164 Primeros auxilios básicos. La información recogida permitió determinar que la necesidades de capacitación que se presentaron en los emprendedores, es debido a que pretenden un mayor comprensión sobre las 
seis áreas de la empresa (administración, recursos humanos, producción, finanzas, servicio al cliente y mercadotecnia), además del resto de temas importantes en la actividad comercial y empresarial, actualizaciones de las dinámicas del entorno social y económico, que por la globalización se convierten en temas de interés para los microempresarios, con la finalidad de estar bien instruidos y capacitados para tomar conspicuas decisiones y hacer más eficaces sus actividades del día a día.

En resumen, los resultados con respecto a las capacitaciones a la población de emprendedores, un buen número de estos emprendedores, en el orden de 284 de 340 registrados, se les ha impartido talleres, acompañamiento y asesorías de capacitación para mejorar la organización y acoplamiento en los mercados del Tena, conforme se desprende en el siguiente gráfico.

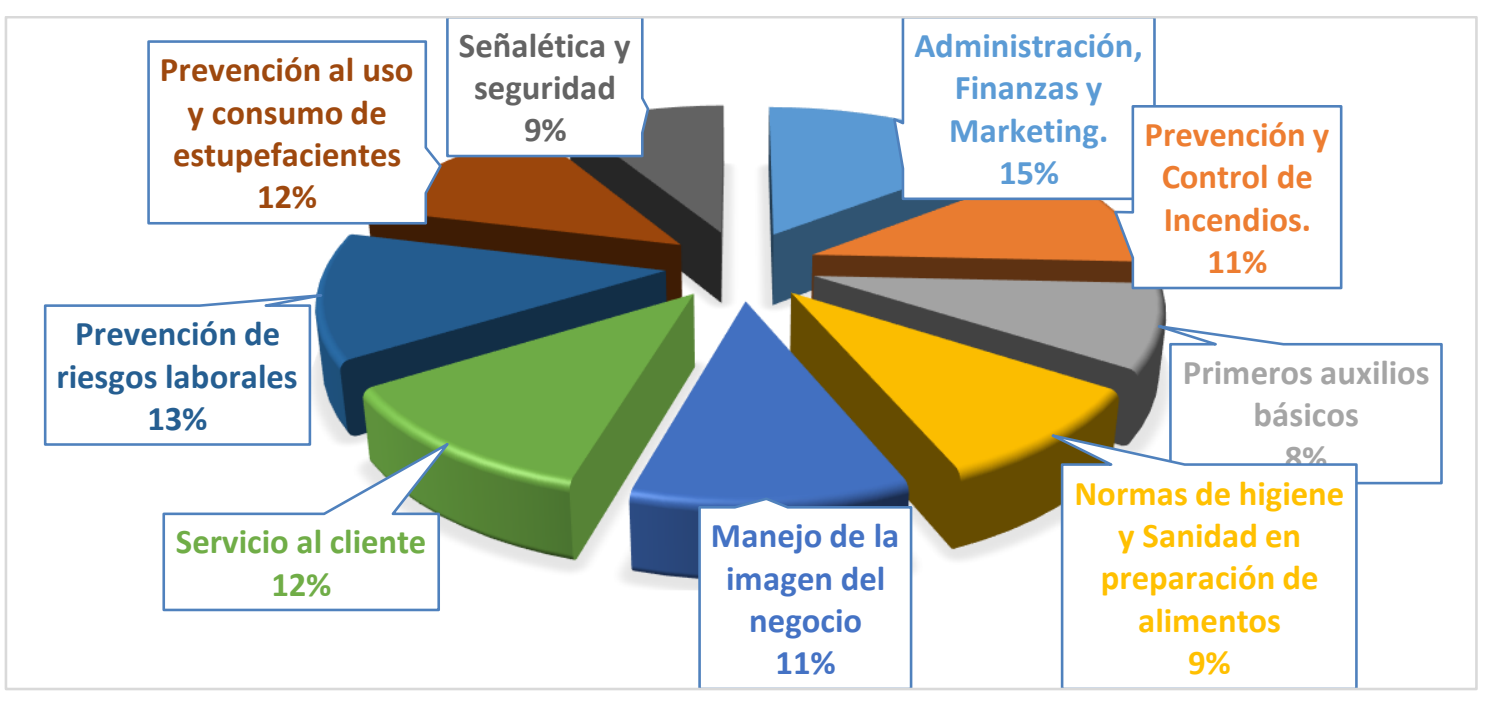

Figura 1. Microempresarios capacitados

Fuente: Proyecto de Vinculación Fortalecimiento al emprendimiento productivo e incubación- Microempresarios de los mercados de la ciudad de Tena en coordinación con EMPUDEPRO.

Elaborado: Grupo Investigador.

Los resultados evidencian porcentualmente que se ha asesorado mediante acompañamientos, en un $15 \%$ de microempresarios, mediante sesiones de trabajo y talleres en temas referentes a la administración, producción, recursos humanaos, control de calidad, investigación de mercados, servicio al cliente, marketing y otros, con el 13\%, salud ocupacional, el 12\% prevención al uso y consumo de sustancias en espacios laborables, el 12\% servicio al cliente, el 11\% prevención y control de incendios, el $11 \%$ manejo de la imagen del negocio, $9 \%$ en normas de higiene y 
ISSN: 2602-8506

Vol. 3, N4., p.20-43, octubre - diciembre, 2019

sanidad en preparación de alimentos, con el $9 \%$ señalética y seguridad, y con el $8 \%$ primeros auxilios básicos, este tipo de actos académicos permitieron mejorar el servicio a los emprendedores y comerciantes, a través de la realización de eventos de capacitación sin costo alguno, permitiendo de esta manera una formación empresarial, de manera que permita un Impulso a la productividad y competitividad de los emprendedores de la ciudad del tena para lograr un crecimiento económico y mejorar las condiciones de vida.

La aplicación de las encuestas a los emprendedores en un total de 148, permitió también realizar el levantamiento de un diagnóstico en la determinación de las actividades económicas de los emprendedores de los mercados.

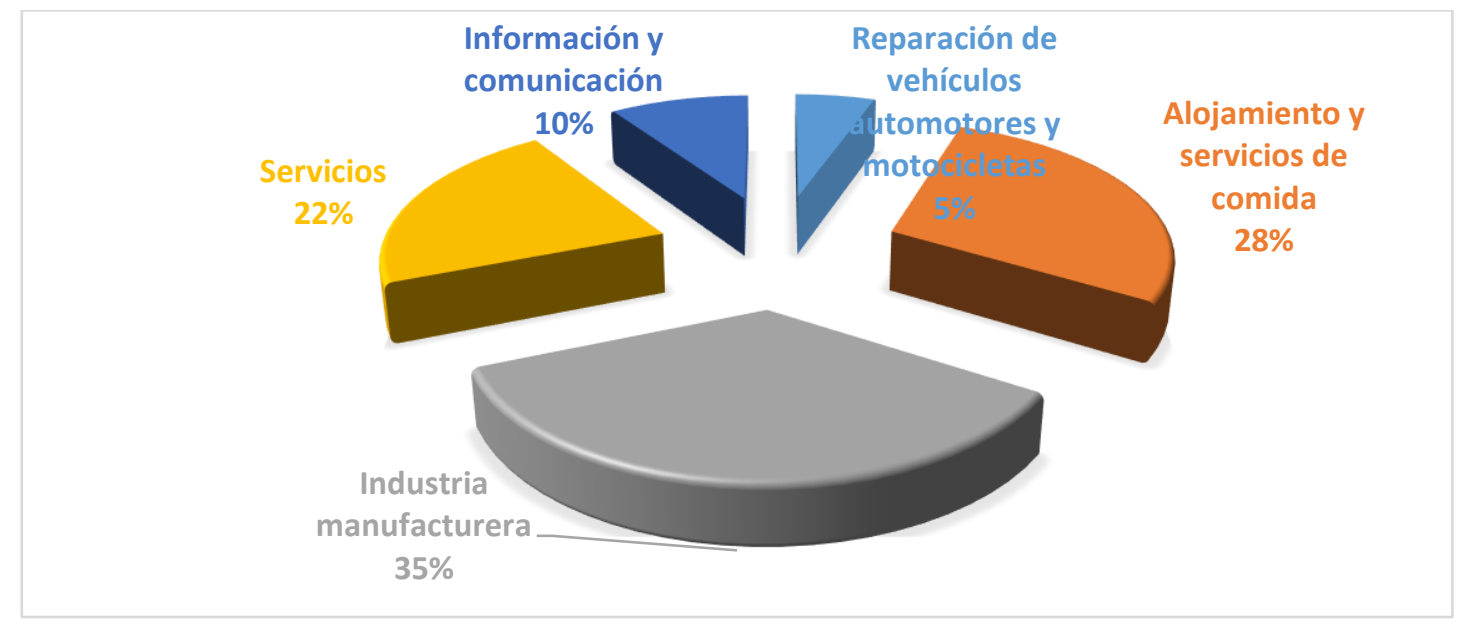

Figura 2. Actividades Económicas de los Emprendedores del Cantón el Tena,

Provincia de Napo, República del Ecuador, Según el CIIU.

Fuente: Investigación propia.

Elaborado: Grupo Investigador.

El análisis indica, que de acuerdo CIIU (Clasificador nacional de actividades económicas), en función de la actividad económica principal se determinó que el 35\% corresponde a la industria manufacturera y artesanías, el $28 \%$ a alojamiento y servicios de comida, el $22 \%$ a servicios (Asesorías por profesionales, escuela de baile, agencia de limpieza, servicio de SPA, Gimnasios, reparación de electrodomésticos, computadoras, alquiler de ropa y etiqueta, parqueo, entre otros), el 9\% corresponde a información y comunicación y el 5\% a la venta de accesorios, repuestos y reparación de vehículos. 
ISSN: 2602-8506

Vol. 3, N4., p.20-43, octubre - diciembre, 2019

Como es lógico, la alta demanda de artesanías y bienes referentes a tejidos, bisutería, collares y demás prendas elaboradas por emprendedores, sumado a esto la deliciosa gastronomía propia de la región amazónica y de la provincia de Napo, son delicias apetecidas por propios e ilustres visitantes.

Así mismo, dentro de los resultados se analizaron el plan de vida ocupacional de los empresarios encuestados.

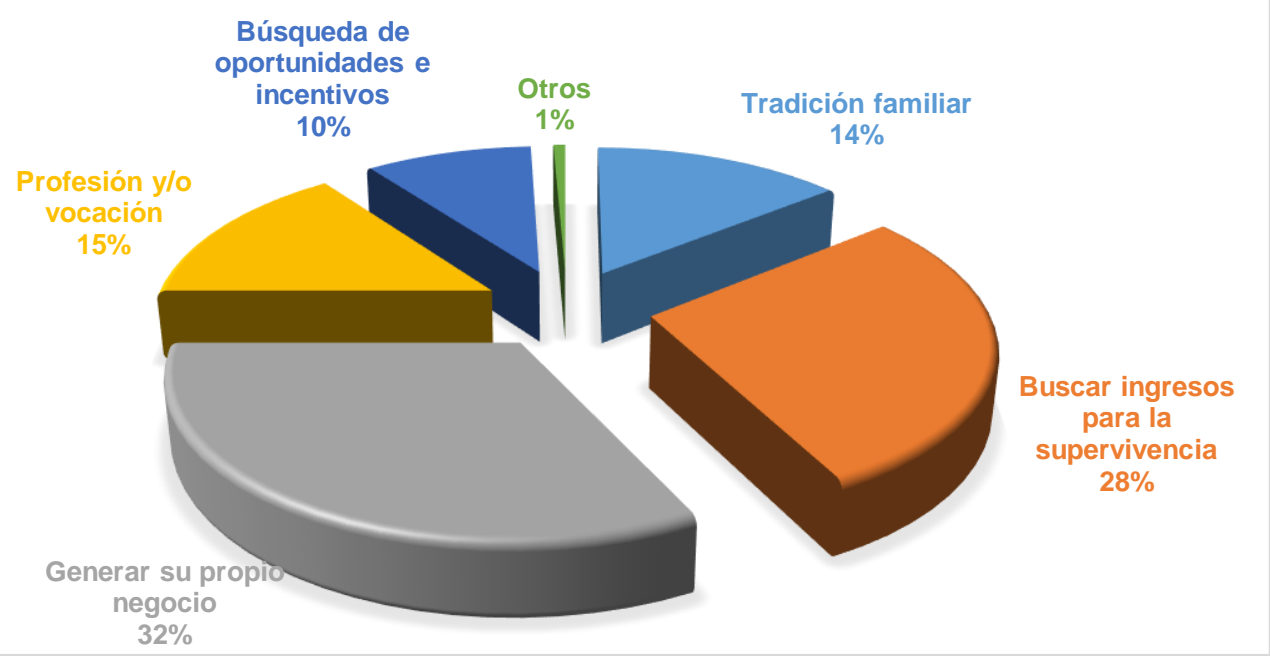

Figura 3. Plan de vida ocupacional

Fuente: Investigación propia.

Elaborado: Grupo Investigador.

Los resultados evidencian que dentro del plan de vida ocupacional los emprendedores en un $32 \%$ decidieron generar su propio negocio, para buscar ingresos para su supervivencia el 28\%; el 15\% consideraron su profesión y/o vocación, el 14\% emprendieron por tradición familiar y el $1 \%$ por otras circunstancias, de allí que sostienen que cada persona que quiera crear su propia empresa es libre de hacerlo, por ello para tener mayores posibilidades de éxito es conveniente tener ciertas competencias y habilidades empezando por la iniciativa, capacidad de tomar decisiones, proactividad, asumir riesgos, autoestima, persistencia, capacidades y motivación, organización, comunicación, conocimientos y formación en gestión empresarial o en la propia actividad que va a desarrollar el emprendimiento, terminando con la buena práctica de valores 
ISSN: 2602-8506

Vol. 3, N4., p.20-43, octubre - diciembre, 2019

tales como la ambición, visión de futuro, determinación, curiosidad, entre otros, y para ello será necesario siempre contar con la transferencia del conocimiento.

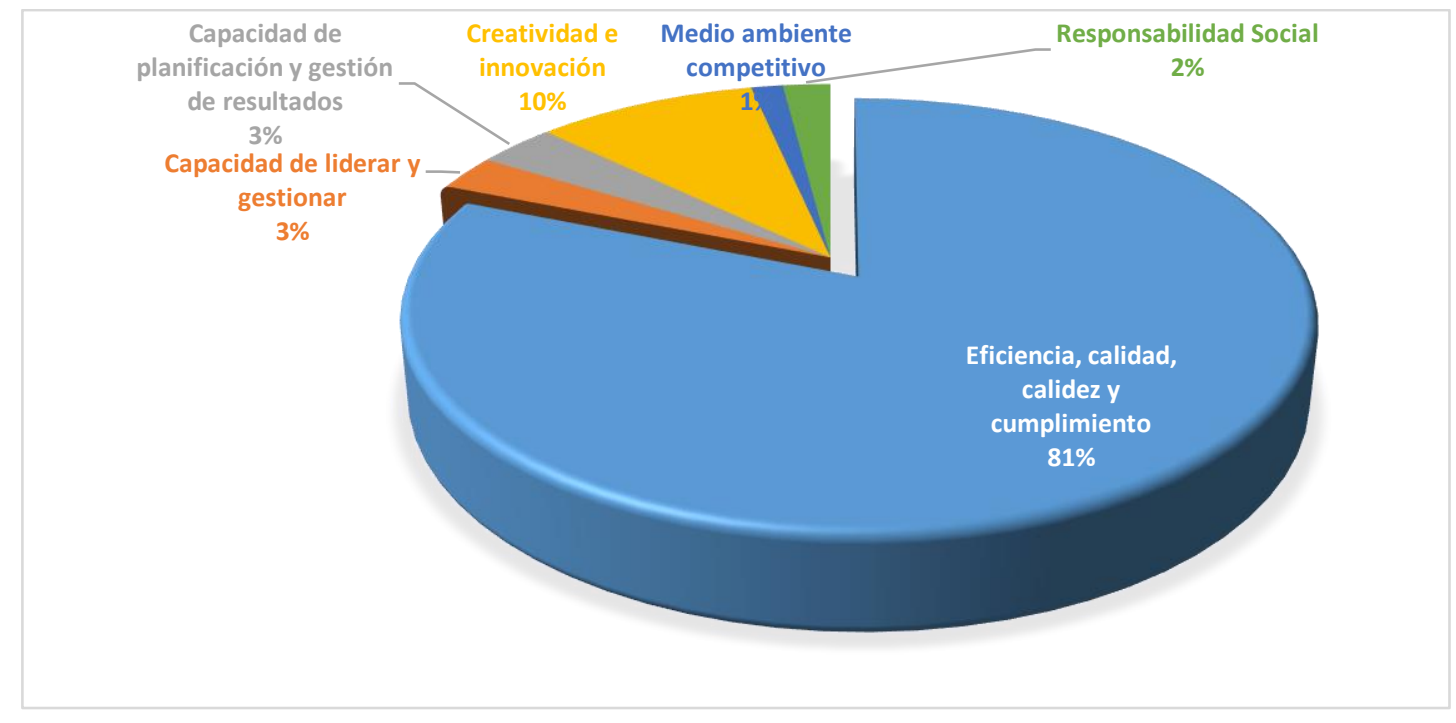

Figura 4. Impacto de las asesorías y capacitaciones

Fuente: Investigación propia.

Elaborado: Grupo Investigador.

La información recogida permite determinar que entre factores que influyen en el emprendimiento, el $81 \%$ de los comerciantes emprendedores están conformes por que han logrado eficiencia, calidad, calidez y cumplimiento tanto en sus actividades micro empresariales, como con sus clientes, el 9\% han logrado creatividad e innovación, el 3\% capacidad de planificación y gestión de resultados, así mismo el 3\% capacidad de liderar y gestionar, el $2 \%$ responsabilidad social y el $1 \%$ medio ambiente competitivo, el análisis, por tanto determina que las competencias y habilidades de los emprendedores mejoró notablemente, por medio de la eficiencia cualquier micro empresario estará en condiciones de prestar servicios o fabricar productos, cumpliendo sus objetivos con el mínimo esfuerzo, gasto o desperdicio, indudablemente que ello le permite reducir sus costos y mejorar los resultados micro empresariales.

Desde el punto de vista de la calidad de un producto o servicio, es la percepción que el cliente tiene del mismo, por consiguiente, es una fijación mental del consumidor que asume conformidad con dicho producto o servicio y la capacidad del mismo para satisfacer sus 
ISSN: 2602-8506

Vol. 3, N4., p.20-43, octubre - diciembre, 2019

necesidades, por otro lado, cuando nos referimos a calidez, nos concentramos en el trato que recibe un cliente por parte de la persona que lo atiende. Un trato agradable, la buena predisposición para escucharlo y entender sus problemas, inquietudes y necesidades, en definitiva, es importante hacerlo sentir "en casa" al cliente, de allí que la calidad y calidez van de la mana, por tales razones la calidez abre las puertas a la calidad. Por otro lado, el cumplimiento refiere a la acción y efecto de cumplir con determinada cuestión o con alguien, por consiguiente, el cumplimiento marcará de alguna manera el camino del éxito o no.

Por último, con respeto a la creatividad e innovación, Coyne (2015), sostiene que "La creatividad es pensar en ideas nuevas y apropiadas, mientras que la innovación es la aplicación con éxito de las ideas dentro de una organización o emprendimiento”. Dicho en otras palabras, las ideas y conocimientos que producen creatividad, conducen a la innovación o procesos, de allí, que dentro de las formas de impulsar o estimular la creatividad encontramos el hecho de motivar, proponer retos, fomentar una participación activa, tener mayor autonomía, por otro lado, dentro de la práctica de la innovación localizamos la generación de ideas para innovar, mediante el impulso de la creatividad, estar atento a los cambios del mercado, lanzamiento de nuevos productos.

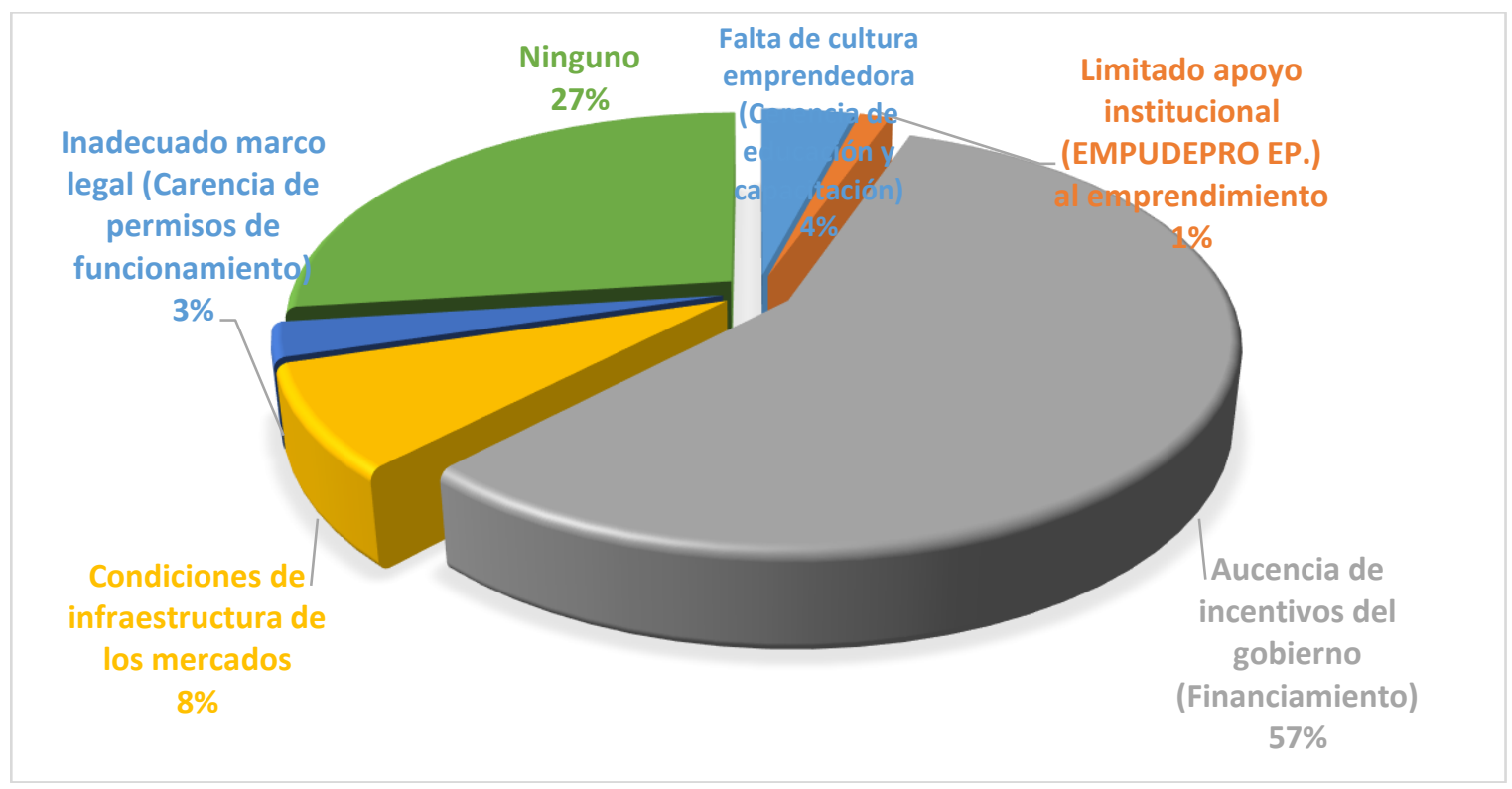

Figura 5. Factores que obstaculizan la sostenibilidad

Fuente: Investigación propia.

Elaborado: Grupo Investigador. 
ISSN: 2602-8506

Vol. 3, $\mathrm{N}^{\circ} 4 .$, p.20-43, octubre - diciembre, 2019

Los resultados evidencian, que los microempresarios consideran que los factores que podrían influir en la sostenibilidad de sus emprendimientos corresponde al 57\%, por ausencia de incentivos del gobierno, es decir la falta de dotación de alicientes tales como capital semilla, bono de desarrollo humano, en temas de emprendimiento, por un lado y por otro lado la dotación de préstamos con intereses bajos y accesibles, con el $27 \%$ revelan que no existirá ningún obstáculo, que sus emprendimientos consideran son sustentables y sostenibles en el tiempo, el $8 \%$ considera que existe problemas de infraestructura en los mercados, mayor énfasis del municipio del Tena en las baterías sanitarias, el $4 \%$ manifiesta que la falta de una constante capacitación y educación, es decir una continua actualización y transferencia del conocimiento, el $3 \%$ la carencia de permisos de funcionamiento y el $1 \%$ un limitado apoyo de la empresa municipal Empudepro.

\section{Discusión}

Como es lógico, el análisis indicó que tanto los micro empresarios como los pequeños empresarios, diseñados bajo la premisa de emprendimientos se han instaurado en los mercados de la ciudad del Tena, a partir de las ideas propias, considerando su lógica, cultura, espíritu emprendedor e interés, emprendimientos totalmente independientes, mismos que se les categoriza dentro del mercado del comercio, en este aspecto son consideradas como los principales motores de empleo trabajo digno, capaz de contribuir con la matriz productiva nacional.

Con respecto a la personalización de los emprendedores que desarrollan sus actividades comerciales en los mercados de la ciudad del Tena, se determinó que la capacitación de los micro empresarios en temas de formación empresarial fue de suma importancia, toda vez que les permitió en un $81 \%$ de conformidad al instrumento de recolección de información, mejorar la eficiencia, calidad, calidez y cumplimiento, siendo sustancial para los emprendedores la elaboración de un plan de negocios como una herramienta de gestión para asegurar su planificación, por consiguiente es necesario trabajar en el fortalecimiento de cadenas productivas para potenciar la producción con alta intensidad tecnológica y valor agregado incorporado, para generar trabajo a través del fortalecimiento de la economía social del conocimiento y la creación de ecosistemas de innovación y emprendimiento, y para la vinculación de la estrategia productiva con la política activa de empleo (PNTV, 2017 p.73) 
ISSN: 2602-8506

Vol. 3, N4., p.20-43, octubre - diciembre, 2019

La Universidad provee datos, estudios y propicias acciones, que se transforman en complementos ideales del trabajo para emprendedores, empresas e instituciones públicas y privadas. En la actualidad resulta oportuno, plantear la relación entre la Universidad y la sociedad, pues de esta manera se puede evidenciar que la Universidad tiene una función social, que influye y transforma su entorno, es decir, es un auténtico factor de transformación social, y, por lo tanto, según sea el fin que se proponga, ayudará a construir una sociedad con determinadas características y competencias, Rojas, B., \& Elena, D. (2017).

De la misma manera con respecto a la sostenibilidad, por supuesto que los factores determinantes en la sostenibilidad de los emprendimientos, varían dependiendo del contexto, el tipo de microempresa, la región geográfica objeto de estudio, el ecosistema de emprendimiento y la coyuntura política y económica, es por ello que el $57 \%$ de los encuestados sustentan que existe ausencia de incentivos del gobierno, es decir apoyo al emprendedor mediante políticas públicas, creando un ecosistema favorable para su actividad económica que fomente su desarrollo y crecimiento en el tiempo; es decir carencia de fondos de inversión pública, entre ellos la falta de dotación de capital semilla, sin generación de intereses, inversión ángel, bono de desarrollo humano, y la dotación de préstamos de las instituciones financieras estatales como BanEcuador, Banco del Pacifico, Corporación Financiera Nacional, entre otros, a intereses bajos para actividades puramente de emprendimientos, A pesar de estas limitaciones, el emprendimiento se debe instituir sobre oportunidades claramente identificadas que den cuenta de la necesidad real y el potencial del mercado. De igual forma, los emprendedores deben identificar sectores económicos cuyo ciclo vital este en pleno crecimiento y localizaciones geográficas que presenten expectativas de crecimiento atractivas para garantizar la demanda de los usuarios de los bienes y servicios.

Por último, los emprendimientos en su fase de creación presentan restricciones estructurales, es por ello que están sujetos a situaciones de riesgo, esto puede deberse a altos niveles de endeudamiento que se traducen en costos financieros descomunales, que impiden planear estratégicamente su crecimiento, si consideramos la diversificación de la oferta como la creación de nuevas unidades de negocios, de allí la importancia que el Gobierno Nacional promueva la 
generación de la inversión de riesgo, proveniente del sector privado, considerando políticas públicas.

\section{Conclusiones}

Se puede concluir que la gran mayoría de las encuestas fueron aplicadas a microempresarios que tienen sus emprendimientos en los mercados del Tena, en donde el $81 \%$, ha podido valorar que en la transferencia de conocimiento está implícito en el concepto productividad, como una de las metas principales a lograr, que implica eficiencia (productividad favorable), eficacia (grado en que el producto o servicio satisface las necesidades reales y potenciales o expectativas de los clientes o usuarios), y efectividad (grado de cumplimiento de los objetivos planificados), por su parte cuando nos referimos a calidez, nos concentramos en el trato que recibe un cliente y/o usuario por parte de la persona que lo atiende. Un trato cordial y afable, igualmente, la calidad es el brindar un excelente servicio al cliente, pues este es la clave para atraer mayor cantidad de clientes, por consiguiente, los clientes busaca momentos y experiencias agradables en los lugares donde adquieren un producto o servicio.

En el caso de las entidades de impulso y fomento gubernamental, es importante consolidar una nueva ley orgánica de emprendimiento e innovación, como base del desarrollo productivo del Ecuador, en donde se establezca un marco normativo que incentive y fomente la creación, desarrollo, crecimiento y expansión de proyectos de emprendimiento a nivel nacional, el desarrollo de las políticas de apoyo al emprendimiento, deben considerar los factores que son determinantes en la sostenibilidad de los emprendimientos, para tal fin debe crearse un vínculo del sistema educativo en todos sus niveles y sistema productivo nacional, mediante la formación en competencias laborales, ciudadanas y empresariales a través de una catedra transversal de emprendimiento.

La presente investigación abordada, es resultado de una serie de vivencias en las calles y mercados de la ciudad del Tena, así como el fruto de encuestas, entrevistas y conversatorios con los involucrados, el mismo que está sujeto a la recepción de nuevos criterios con nuevas investigaciones que permitan cada vez sentar nuevas propuestas que garanticen un mejor ordenamiento de la ciudad y se disminuya los inconvenientes y dificultades que son ocasionados por la ciudadanía y los visitantes sean propios y/o extraños. 
Vol. 3, N4., p.20-43, octubre - diciembre, 2019

Con respecto a los temas de acompañamiento, talleres, asesorías y sesiones de trabajo realizados, a la mayoría de los arrendatarios del mercado central, mercado sur (feria libre), mercado de Misahuallí; y patio de comidas "Eloy Alfaro", han manifestado estar satisfechos con los temas tratados e impartidos por estudiantes de la UCACUE, por cuanto son importantes para mejorar su atención a los clientes, entregando productos sanos y de calidad, además de llevar de manera organizada sus operaciones administrativas, contables, financieras y tributarias.

Los resultados evidencian la vinculación del modelo triple hélice en la relación UniversidadEmpresa-Gobierno, por consiguiente, existió la integración de diferentes actores, en donde los agentes y organizaciones se comunican, cooperan y establecen relaciones de corto, mediano y largo plazo y condiciones económicas, jurídicas y tecnológicas para el fortalecimiento del emprendimiento, la innovación y la productividad de este sector de la República del Ecuador.

La información recogida permitió determinar la presencia del comercio informal, mismo que se manifestó para subsistir ante la amenaza del desempleo causado por la marginación social por determinantes como la edad, habilidades laborales o factores familiares y educación cuyo modelo perjudica a la economía local, presentando obstáculos para una mejor organización y acoplamiento, ocasionando dificultades que provoca problemas en el mercado laboral.

Con respecto a los temas de capacitación que han sido considerados más importantes y que han tenido buena aceptación de acuerdo a la revisión bibliográfica y documental se estableció en un $15 \%$ de microempresarios, mediante sesiones de trabajo y talleres en temas referentes a la administración, producción, recursos humanaos, control de calidad, investigación de mercados y marketing, 13\% salud ocupacional, $12 \%$ prevención al uso y consumo de sustancias en espacios laborables, $12 \%$ servicio al cliente, $11 \%$ prevención y control de incendios, $11 \%$ manejo de la imagen del negocio, $9 \%$ en normas de higiene y sanidad en preparación de alimentos, 9\% Señalética y seguridad; y, 8\% primeros auxilios básicos, este tipo de transferencia del conocimiento permitieron fortalecer la cultura emprendedora y mejorar el servicio a los consumidores. 
Vol. 3, N4., p.20-43, octubre - diciembre, 2019

Otro aspecto que constituye de fundamental importancia es que todos los comerciantes ambulantes (informales), se encuentren ubicados en un lugar fijo específico, dando una imagen de negocios sólidos, dirigidos por comerciantes capacitados y conocedores de la actividad que se encuentran realizando.

Es visible el papel que realiza la empresa pública de desarrollo productivo y competitividad, "Empudepro-Tena EP", por cuanto mediante el proyecto de vinculación se destaca el desarrollo de la cultura emprendedora en los estudiantes de la Universidad Católica de Cuenca, al momento de promover estrategias hacia la generación de empleo para la supervivencia de los pequeños comerciantes, espacios de trabajo, debidamente acondicionados con todos los servicios básicos que se requieren para expedir y prestar servicios de calidad, con entusiasmo y decisión, para que los clientes se sientan satisfechos y contentos por sus adquisiciones que le permitirán satisfacer plenamente las necesidades que tienen.

Es conveniente destacar también que gracias a la participación en los cursos y talleres los comerciantes tanto formales como informales en un numero de 284 lograron unos actualizar sus conocimientos en temas descritos en el cuadro No 1 y otros regularizarse, mediante la entrega de los permisos de funcionamiento, otorgados por la empresa pública "Empudepro-Tena EP", como es lógico los acompañamiento, talleres, asesorías y resultados de sesiones de trabajo, fueron determinantes para mejorar la organización y lograr un mejor ordenamiento de los mercados.

El análisis indico que se han cumplido en parte con los postulados del Plan Nacional de Desarrollo Todo una Vida, con respecto a la generación de empleo en este hermoso sector del Oriente Ecuatoriano, de allí que se han aplicado políticas públicas para mejorar las condiciones de trabajo y fortalecer el empleo, pero como es obvio aún queda mucho por hacer, por ello será importante seguir promoviendo trabajo en conjunto entre el Gobierno, empresa y el aporte de la Universidad. Es evidente que el resultado periódico de las actividades de los emprendedores, les proporciona bienestar, alegría por lo que realizan, mejorando los niveles de calidad de vida y contribuyendo al crecimiento del sector. 
Vol. 3, N4., p.20-43, octubre - diciembre, 2019

La información recogida permite determinar que los habitantes de la ciudad del Tena están orgullosos de vivir en una ciudad que muestra que sus autoridades están empeñadas en una administración eficiente en bien de todos, con un correcto ordenamiento territorial y respeto por la normativa que regula el comercio.

La Empresa Pública de Desarrollo Productivo y Competitividad "Empudepro Tena-EP”, debe continuar dando la apertura y facilidades, para que otros Estudiantes de distintas Universidades, tengan la oportunidad de seguir conociendo de cerca el funcionamiento, administración, los retos y modelo de éxito que impulsa la creación de las Empresas Públicas del Estado, esta inclusión permitirá involucrarse y en un futuro también poder contribuir con el progreso y desarrollo, que hoy en día es vital para la dinamización de las economías locales.

\section{Referencias bibliográficas.}

Agenda 2030 y los Objetivos de Desarrollo Sostenible, (2016). Una oportunidad para América Latina y el Caribe, Publicación de las Naciones Unidas, mayo de 2016, Impreso en Santiago.

Argos, M. J. (2016). El comercio informal y su incidencia en las ventas de los comerciantes formales del centro comercial popular "La Condamine" sección prendas de vestir durante el periodo 2014. Tesis, Universidad Nacional de Chimborazo, Riobamba, Ecuador. Obtenido de http://dspace.unach.edu.ec/bitstream/51000/3887/1/UNACH-ECFCP-INGCOM-2017-0016.pdf.

Arieu, A. 2012. La Educación de los emprendedores: Un comentario acerca de los contenidos formativos y el rol de la Universidad. Congreso Provincial Emprendedorismo y Desarrollo Local. Pigüe, Argentina.

Alkire, S. \& Foster, J. (2008). Recuento y Medición Multidimensional de la Pobreza:

Recuperado de: https://ophi.org.uk/recuento-y-medicion-multidimensional-de-lapobreza

Banco Mundial (2014), América latina en movimiento, recuperado de: https://www.alainet.org/es/articulo/173175.

Global Entrepreneurship Monitor GEM Ecuador (2014), recuperado de https://www.revistalideres.ec/lideres/ecuador-lidera-tasa-emprendimientonecesidad.html

Lituma, N. (2017). Centro comercial el Arenal. Tesis de Maestría, Universidad Estatal, Cuenca, Ecuador.

Pacek, N. y. (2008). Oportunidades en los Mercados Emergentes. Colección Finanzas y Negocios.

Rojas, B., \& Elena, D. (2017). Análisis del impacto en el sector laboral de la ciudad de 
ISSN: 2602-8506

Vol. 3, N4., p.20-43, octubre - diciembre, 2019

Esmeraldas de las prácticas pre profesionales de los estudiantes de las carreras administrativas de la PUCESE del período 2016-2017. ESMERALDAS.

Romero Reyes, K. S. (agosto de 2010). La capacitación y la motivación como herramientas para conseguir una ventaja competitiva. Recuperado de http://cdigital.uv.mx/bitstream/123456789/28840/1/Romero\%20Reyes.pdf

Werther, W., \& Davis, K. (2011). Administración de personal y recursos humanos. Recuperado de:

https://jgestiondeltalentohumano.files.wordpress.com/2013/11/administracion-depersonal-y-recursos-humanos-tercera-edicion-william-werther-keith-davis.pdf

Zamora, G., X. Villamar, 2011. Caracterización de la PYME en la Industria Manufacturera del Distrito Metropolitano de Quito. Centro de Publicaciones de la Pontificia Universidad Católica del Ecuador (PUCE), Quito, Ecuador.

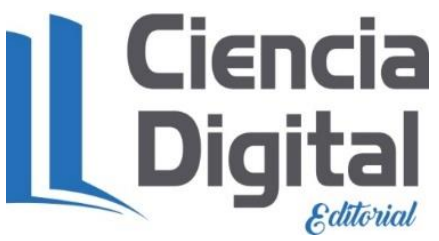




\section{PARA CITAR EL ARTÍCULO INDEXADO}

Becerra Molina, E., Ramón Poma, G., Venegas Sánchez, E., \& Astudillo Arias, P. (2019). Impulso a la productividad y competitividad de los emprendedores de la ciudad del Tena para el crecimiento económico sustentable. Visionario Digital, 3(4), 20-43.

https://doi.org/10.33262/visionariodigital.v3i4.950

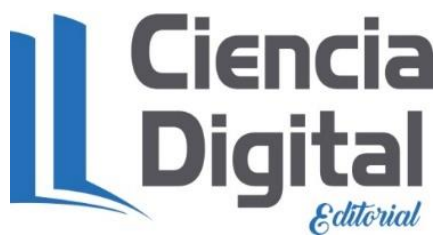

El artículo que se publica es de exclusiva responsabilidad de los autores y no necesariamente reflejan el pensamiento de la Revista Visionario Digital.

El artículo queda en propiedad de la revista y, por tanto, su publicación parcial y/o total en otro medio tiene que ser autorizado por el director de la Revista Visionario Digital.
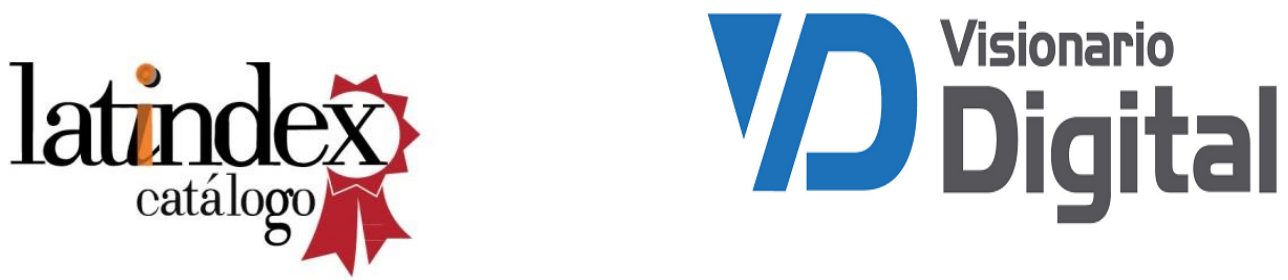